\title{
Property of oscillation of first order impulsive neutral differential equations with positive and negative coefficients
}

\author{
Hussain Ali Mohamad \\ Department of Mathematics \\ College of science for Women
}

Aqeel Falih Jaddoa

Department of Mathematics

College of Education for Pure Science

Ibn Al-Haitham

\section{University of Baghdad, Iraq}

Recived : 10\10\2018

Revised : 23\10\2018

Accepted : $5 \backslash 11 \backslash 2018$

Available online : $\quad 24 / 1 / 2019$

DOI: $10.29304 / j q c m .2019 .11 .1 .457$

\begin{abstract}
.
In this paper, necessary and sufficient conditions for oscillation are obtained, so that every solution of the linear impulsive neutral differential equation with variable delays and variable coefficients oscillates. Most of authors who study the oscillatory criteria of impulsive neutral differential equations, investigate the case of constant delays and variable coefficients. However the points of impulsive in this paper are more general. An illustrate example is given to demonstrate our claim and explain the results.
\end{abstract}

Keywords: Oscillation, Impulses effect, Neutral differential equations.

Mathematics Subject Classification: 34K11. 


\section{INTRODUTION}

The investigation in the theory of impulsive differential equations is now not only wider than the theory of differential equations without impulses effect, but it describes many phenomena and processes more reality so it has a lot of applications in many natural and industrial fields to study different characters and it can used as a tool in mathematical models for instance, in medicine [1], control theory [2], population dynamics [3], in neural networks [4] and etc. In fact, many evolution processes are often developed for immediate perturbations and sudden changes in specific moments of time such as in biological system in heart beats. This period of change is very small compared to the periods of operation, therefore the situation is quite different from what it is in differential equations without impulses in many physical phenomena, and it appears as a sudden change in its state. The consideration of oscillatory solutions for impulsive neutral differential equations is a new and wide object to find the qualitative properties. There is a lot of research and monographs that deal with the conditions to guarantee the oscillation of all solutions the impulsive neutral differential equations with coefficients such as variable coefficients and constant delays see $[5,6]$. There are some results of oscillation for this type of equations [7-12] and we noted that the search of oscillation with impulsive neutral differential equations is more difficult than the type without it. Shen etc. al [13-17] obtained sufficient conditions for oscillation of all solutions of first order impulsive neutral differential equation of constant delay with positive and negative coefficients are obtained. Consider neutral differential equation:

$$
\left.\begin{array}{c}
{[y(t)-P(t) y(\tau(t))]^{\prime}+Q(t) y(\sigma(t))-R(t) y(\alpha(t))=0,} \\
t \neq t_{k} \quad k=1,2, \ldots \\
y\left(t_{k}^{+}\right)+b_{k} y\left(t_{k}\right)=a_{k} y\left(t_{k}\right), \quad t=t_{k} \quad k=1,2, \ldots
\end{array}\right\}
$$

Where $P \in P C\left(\left[t_{0}, \infty\right) ; R^{+}\right)$and $Q, R, \in$

$C\left(\left[t_{0}, \infty\right) ; R^{+}\right), \quad$ and $\quad \tau(t), \alpha(t), \sigma(t) \in$ $C\left(\left[t_{0}, \infty\right) ; R\right), \quad \lim _{t \rightarrow \infty} \tau(t)=\infty, \lim _{t \rightarrow \infty} \alpha(t)=$ $\infty, \lim _{t \rightarrow \infty} \sigma(t)=\infty$ where $\tau, \alpha, \sigma$ are increasing functions. The functions $\tau^{-1}(t), \sigma^{-1}(t), \alpha^{-1}(t)$ are the inverse of the functions $\tau(t), \alpha(t), \sigma(t)$ respectively.

\section{SOME BASIC LEMMAS}

The following lemmas will be useful in the proof of our main results: Throughout the paper we assume that $\tau(t), \alpha(t), \sigma(t)<t$, for $t \in$ $\left(t_{k}, t_{k+1}\right], t_{1} \geq t_{0}, k=1,2, \ldots$

Lemma 2.1. [^] Suppose that $g, h \in C\left(R^{+}, R^{+}\right)$, $h(t)<t$ for $t \geq t_{0}$, $\lim _{t \rightarrow \infty} h(t)=\infty$ and

$$
\liminf _{t \rightarrow \infty} \int_{h(t)}^{t} g(s) d s>\frac{1}{e}
$$

then the inequality $y^{\prime}(t)+g(t) y(h(t)) \leq 0$ has no eventually positive solutions.

Lemma 2.2. Let $y(t)$ be an eventually positive solution of equation (1.1) and there exists a continuous function $\delta(t)$ such that:

$W(t)$

$=y(t)-P(t) y(\tau(t))-\int_{\alpha^{-1}(\delta(t))}^{t} R(u) y(\alpha(u)) d u$

$-\int_{t}^{\sigma^{-1}(\delta(t))} Q(u) y(\sigma(u)) d u$

Where $\delta(t)<t$ and $t \in\left(t_{k}, t_{k+1}\right], 0<t_{0}<t_{1}<$ $\cdots<t_{k} \rightarrow \infty$ as $k \rightarrow \infty$.

Also $\alpha^{-1}(\delta(t))<t$ and $\sigma^{-1}(\delta(t))>t$, in addition to the following assumptions:

$$
\begin{gathered}
\text { H1: } Q\left(\sigma^{-1}(\delta(t))\right)\left(\sigma^{-1}(\delta(t))\right)^{\prime} \\
-R\left(\alpha^{-1}(\delta(t))\right)\left(\alpha^{-1}(\delta(t))\right)^{\prime} \\
\geq 0, \\
t \in\left(t_{k}, t_{k+1}\right], \quad k=1,2, \ldots
\end{gathered}
$$

H2: There exists two positive real numbers $a_{k}$ and $b_{k}$ such that

$$
\begin{gathered}
0<a_{k}-b_{k} \leq 1, k=1,2, \ldots \text { And } \\
\left\{\begin{array}{c}
P\left(t_{k}^{+}\right) \geq\left(a_{k}-b_{k}\right) P\left(t_{k}\right) \text { for } \tau\left(t_{k}\right) \neq t_{i}, i<k, \\
P\left(t_{k}^{+}\right) \geq \frac{1}{a_{k}-b_{k}} P\left(t_{k}\right) \text { for } \tau\left(t_{k}\right)=t_{i}, i<k,
\end{array}\right.
\end{gathered}
$$

Where $a_{k}=a_{i}, b_{k}=b_{i}$ when $\tau\left(t_{k}\right)=t_{i}, i<k$

H3: $\limsup _{t \rightarrow \infty}\left[P(t)+\int_{\alpha^{-1}(\delta(t))}^{t} R(u) d u\right.$

$$
\begin{aligned}
& \left.+\int_{t}^{\sigma^{-1}(\delta(t))} Q(u) d u\right] \leq 1, \quad t \\
& \quad \in\left(t_{k}, t_{k+1}\right] .
\end{aligned}
$$

Then $W(t)$ is eventually positive and nonincreasing function. 
Hussain .A/Aqeel .F

Proof. Let $y(t)$ be an eventually positive solution of equation(1.1) that is $y(t)>$ $0, y(\tau(t))>0, \quad y(\sigma(t))>0$ and $y(\alpha(t))>0$, $t \geq t_{0}$,

Differentiate (2.2) for every interval $\left(t_{k}, t_{k+1}\right]$ where $\mathrm{k}=1,2, \ldots$ and use (1.1) we get

$W^{\prime}(t)$

$=[y(t)-P(t) y(\tau(t))]^{\prime}-R(t) y(\alpha(t))$

$+R\left(\alpha^{-1}(\delta(t))\right) y(\delta(t))\left(\alpha^{-1}(\delta(t))\right)^{\prime}$

$-Q\left(\sigma^{-1}(\delta(t))\right) y(\delta(t))\left(\sigma^{-1}(\delta(t))\right)^{\prime}$

$+Q(t) y(\sigma(t))$

$$
\begin{aligned}
= & -Q(t) y(\sigma(t))+R(t) y(\alpha(t)) \\
& -R(t) y(\alpha(t)) \\
+ & R\left(\alpha^{-1}(\delta(t))\right) y(\delta(t))\left(\alpha^{-1}(\delta(t))\right)^{\prime} \\
- & Q\left(\sigma^{-1}(\delta(t))\right) y(\delta(t))\left(\sigma^{-1}(\delta(t))\right)^{\prime} \\
+ & Q(t) y(\sigma(t)) \\
= & -\left[Q\left(\sigma^{-1}(\delta(t))\right)\left(\sigma^{-1}(\delta(t))\right)^{\prime}\right. \\
- & \left.R\left(\alpha^{-1}(\delta(t))\right)\left(\alpha^{-1}(\delta(t))\right)^{\prime}\right] y(\delta(t)) \\
\leq & 0(2.3)
\end{aligned}
$$

Hence $W(t)$ is nonincreasing function on each $t \in\left(t_{k}, t_{k+1}\right]$ for $k=1,2, \ldots$

To prove that $W\left(t_{k}^{+}\right) \leq W\left(t_{k}\right)$ for $\mathrm{k}=1,2, \ldots$ In view of $0<a_{k}-b_{k} \leq 1, k=1,2, \ldots$, we have $0<a_{k}-b_{k} \leq 1$ and from (2.2) with regard to the condition $\boldsymbol{H} 2$ when $\tau\left(t_{k}\right)=t_{i}, i<k$, then:

$$
\begin{aligned}
W\left(t_{k}^{+}\right)=\left(a_{k}-\right. & \left.b_{k}\right) y\left(t_{k}\right)-P\left(t_{k}^{+}\right)\left(a_{k}\right. \\
& \left.-b_{k}\right) y\left(\tau\left(t_{k}\right)\right) \\
& -\int_{\alpha^{-1}\left(\delta\left(t_{k}\right)\right)}^{t_{k}} R(u) y(\alpha(u)) d u \\
& -\int_{t_{k}}^{\sigma^{-1}\left(\delta\left(t_{k}\right)\right)} Q(u) y(\sigma(u)) d u
\end{aligned}
$$

$\leq$

$y\left(t_{k}\right)-P\left(t_{k}\right) y\left(\tau\left(t_{k}\right)\right)-$

$\int_{\alpha^{-1}\left(\delta\left(t_{k}\right)\right)}^{t_{k}} R(u) y(\alpha(u)) d u$

$-\int_{t_{k}}^{\sigma^{-1}\left(\delta\left(t_{k}\right)\right)} Q(u) y(\sigma(u)) d u$

$=W\left(t_{k}\right)$

When $\tau\left(t_{k}\right) \neq t_{i}, i<k$ then from (2.2) with regard to the condition $\boldsymbol{H 2}$ :

$$
\begin{array}{r}
W\left(t_{k}^{+}\right)=\left(a_{k}-b_{k}\right) y\left(t_{k}\right)-P\left(t_{k}^{+}\right) y\left(\tau\left(t_{k}\right)\right) \\
-\int_{\alpha^{-1}\left(\delta\left(t_{k}\right)\right)}^{t_{k}} R(u) y(\alpha(u)) d u \\
-\int_{t_{k}}^{\sigma^{-1}\left(\delta\left(t_{k}\right)\right)} Q(u) y(\sigma(u)) d u \\
\leq\left(a_{k}-b_{k}\right) y\left(t_{k}\right)-\left(a_{k}-b_{k}\right) P\left(t_{k}\right) y\left(\tau\left(t_{k}\right)\right)
\end{array}
$$

$$
\begin{aligned}
& -\left(a_{k}-b_{k}\right) \int_{\alpha^{-1}\left(\delta\left(t_{k}\right)\right)}^{t_{k}} R(u) y(\alpha(u)) d u \\
& -\left(a_{k}-b_{k}\right) \int_{t_{k}}^{\sigma^{-1}\left(\delta\left(t_{k}\right)\right)} Q(u) y(\sigma(u)) d u \\
& =\left(a_{k}-b_{k}\right) W\left(t_{k}\right) \\
& \leq W\left(t_{k}\right)
\end{aligned}
$$

$W(t)$ is non-increasing on $\left[t_{0}, \infty\right)$. Hence $-\infty \leq L<$ $\infty$. Where $|L|=\sup \left\{W\left(t_{k}^{+}\right), \lim _{k \rightarrow \infty} W\left(t_{k}\right)\right\}$, $t \in\left[t_{l}, \infty\right)$ for some $l \geq t_{0}$. We claim that $W\left(t_{k}\right) \geq$ 0 for $\mathrm{k}=l, l+1, \ldots$. Otherwise there exists some $m \geq l$ such that:

$W\left(t_{m}\right)=-\mu<0, \quad$ implies that $W(t) \leq-\mu$ for $t \geq t_{m}$, since $W(t)$ is non-increasing on $\left[t_{l}, \infty\right)$, then for each $t \in\left(t_{k}, t_{k+1}\right], \mathrm{k}=l, l+$ $1, \ldots$ we get from (2.2): $y(t) \leq-\mu+P(t) y(\tau(t))$

$$
\begin{array}{r}
+\int_{\alpha^{-1}(\delta(t))}^{t} R(u) y(\alpha(u)) d u \\
+\int_{t}^{\sigma^{-1}(\delta(t))} Q(u) y(\sigma(u)) d u .
\end{array}
$$

So, we have two cases to consider:

Case 1. If $y(t)$ is unbounded then there exists a sequence of points $\left\{s_{n}\right\}$ such that

$$
\begin{aligned}
& s_{n} \geq t_{m}, \lim _{n \rightarrow \infty} y\left(s_{n}\right)=\infty \text { and } \\
& y\left(s_{n}\right)=\max \left\{y(t), t_{m} \leq t \leq s_{n}\right\} .
\end{aligned}
$$

Then (2.5) reduce to:

$$
\begin{aligned}
y\left(s_{n}\right) & \leq-\mu+P\left(s_{n}\right) y\left(\tau\left(s_{n}\right)\right) \\
& +\int_{\alpha^{-1}\left(\delta\left(s_{n}\right)\right)}^{s_{n}} R(u) y(\alpha(u)) d u \\
& +\int_{s_{n}}^{\sigma^{-1}\left(\delta\left(s_{n}\right)\right)} Q(u) y(\sigma(u)) d u . \\
\leq & -\mu+\left\{P\left(s_{n}\right)+\int_{\alpha^{-1}\left(\delta\left(s_{n}\right)\right)}^{s_{n}} R(u) d u\right. \\
& \left.+\int_{s_{n}}^{\sigma^{-1}\left(\delta\left(s_{n}\right)\right)} Q(u) d u\right\} y\left(s_{n}\right) \\
\leq & -\mu+y\left(s_{n}\right)
\end{aligned}
$$

Leads to $0 \leq-\mu$ which is a contradiction.

Case 2. If $y(t)$ is bounded that is $\limsup _{t \rightarrow \infty} y(t)=\mathrm{M}<$ $\infty$.We can choose a sequence of points $\left\{s_{n}\right\}$ such that

$\lim _{n \rightarrow \infty} y\left(s_{n}\right)=\mathrm{M}$ and $y\left(\eta\left(s_{\mathrm{n}}\right)\right)=\max \left\{\mathrm{y}(\mathrm{t}): \rho_{1}\left(s_{n}\right) \leq\right.$ $\left.t \leq \rho_{2}\left(s_{n}\right)\right\}$. 
Hussain .A/Aqeel .F

Where $\rho_{1}\left(s_{n}\right)=\min \left\{\tau\left(s_{n}\right), \sigma\left(s_{n}\right)\right\}$, $\rho_{2}\left(s_{n}\right)=\max \left\{\tau\left(s_{n}\right), \alpha\left(s_{n}\right)\right\}$ it is obvious that $\lim _{n \rightarrow \infty} \eta\left(s_{n}\right)=\infty$ and limsup $y\left(\eta\left(s_{n}\right)\right) \leq M$.

$$
\begin{aligned}
y\left(s_{n}\right) \leq & -\mu+P\left(s_{n}\right) y\left(\tau\left(s_{n}\right)\right) \\
& +\int_{\alpha^{-1}\left(\delta\left(s_{n}\right)\right)}^{s_{n}} R(u) y(\alpha(u)) d u \\
& +\int_{s_{n}}^{\sigma^{-1}\left(\delta\left(s_{n}\right)\right)} Q(u) y(\sigma(u)) d u \\
\leq- & \mu+\left\{P\left(s_{n}\right)+\int_{\alpha^{-1}\left(\delta\left(s_{n}\right)\right)}^{s_{n}} R(u) d u\right. \\
& \left.+\int_{s_{n}}^{\sigma^{-1}\left(\delta\left(s_{n}\right)\right)} Q(u) d u\right\} y\left(\eta\left(s_{\mathrm{n}}\right)\right) \\
\leq-\mu & +y\left(\eta\left(s_{\mathrm{n}}\right)\right)
\end{aligned}
$$

Taking the superior limit as $\mathrm{n} \rightarrow \infty$, we get

$\mathrm{M} \leq-\mu+\mathrm{M}$, which is also a contradiction.

Combining the cases 1 and 2 , we see that $W(t) \geq 0$ for $t \in\left(t_{k}, t_{k+1}\right], \mathrm{k}=l, l+1, \ldots$.

Since $W(t)$ is nonincreasing, so $W\left(t_{k}\right) \geq W(t) \geq$ 0 for $t \in\left(t_{k}, t_{k+1}\right]$.

To prove $W(t)>0$, we first prove that $W\left(t_{k}\right)>$ 0 for $k=1,2, \ldots$. If it is not true, then there exists some $\mathrm{m} \geq 0$ such that $W\left(t_{m}\right)=0$, integrating (2.3) on $\left(t_{m}, t_{m+1}\right]$ yield:

$$
\begin{gathered}
W\left(t_{m+1}\right)=W\left(t_{m}^{+}\right) \\
-\int_{t_{m}}^{t_{m+1}}\left[Q\left(\sigma^{-1}(\delta(t))\right)\left(\sigma^{-1}(\delta(t))\right)^{\prime}\right. \\
\left.-R\left(\alpha^{-1}(\delta(t))\right)\left(\alpha^{-1}(\delta(t))\right)^{\prime}\right] y(\delta(t)) d t \\
<W\left(t_{m}^{+}\right) \leq W\left(t_{m}\right)=0
\end{gathered}
$$

This contradiction shows that $W\left(t_{k}\right)>0$ for $k=$ $1,2 \ldots$, as well as $W(t) \geq W\left(t_{k+1}\right)>0$, for $t \in\left(t_{k}, t_{k+1}\right], k=1,2, \ldots$. Thus $W(t)>0$ for $t \geq t_{0}$. The proof is complete.

\section{MAIN RESULTS}

The next results provide sufficient conditions for the oscillation of all solutions of (1.1):

Theorem 3.1. Let $W(t)$ defined as in (2.2) and the assumptions $\boldsymbol{H 1}-\boldsymbol{H} \mathbf{3}$ hold, and there exist a continuous function $\delta(\mathrm{t})<t$ such that

$$
\begin{aligned}
& \liminf _{\mathrm{t} \rightarrow \infty} \int_{\delta(t)}^{\mathrm{t}}\left[Q\left(\sigma^{-1}(\delta(\mathrm{s}))\right)\left(\sigma^{-1}(\delta(\mathrm{s}))\right)^{\prime}\right. \\
& \left.-R\left(\alpha^{-1}(\delta(\mathrm{s}))\right)\left(\alpha^{-1}(\delta(\mathrm{s}))\right)^{\prime}\right][1+P(\delta(\mathrm{s})) \\
& \left.+\int_{\alpha^{-1}(\delta(\delta(\mathrm{s})))}^{\delta(\mathrm{s})} R(u) d u+\int_{\delta(\mathrm{s})}^{\sigma^{-1}(\delta(\delta(\mathrm{s})))} Q(u) d u\right] \mathrm{ds} \\
& >\frac{1}{e}
\end{aligned}
$$

Where $\alpha^{-1}(\delta(t))<t$ and $\sigma^{-1}(\delta(\mathrm{t}))>t$, then every solution of equation (1.1) oscillates.

Proof. Suppose that $y(t)$ is eventually positive solution of (1.1) then by Lemma 2.2 it follows that $W(t)$ is positive nonincreasing function for $t \in$ $\left(t_{k}, t_{k+1}\right], k=1,2, \ldots$, since $W(t) \leq y(t)$, hence it follows from (2.2):

$$
\begin{aligned}
& y(t)=W(t)+P(t) y(\tau(t)) \\
& +\int_{\alpha^{-1}(\delta(t))}^{t} R(u) y(\alpha(u)) d u \\
& +\int_{t}^{\sigma^{-1}(\delta(t))} Q(u) y(\sigma(u)) d u \\
& \geq W(t)+P(t) W(\tau(t)) \\
& +\int_{\alpha^{-1}(\delta(t))}^{t} R(u) W(\alpha(u)) d u \\
& +\int_{t}^{\sigma^{-1}(\delta(t))} Q(u) W(\sigma(u)) d u \\
& \geq W(t)+P(t) W(\tau(t)) \\
& +W(\alpha(t)) \int_{\alpha^{-1}(\delta(t))}^{t} R(u) d u \\
& +W(\delta(t)) \int_{t}^{\sigma^{-1}(\delta(t))} Q(u) d u \\
& \geq W(t)+P(t) W(t) \\
& +W(t) \int_{\alpha^{-1}(\delta(t))}^{t} R(u) d u \\
& +W(t) \int_{t}^{\sigma^{-1}(\delta(t))} Q(u) d u \\
& =W(t)[1+P(t) \\
& +\int_{\alpha^{-1}(\delta(t))}^{t} R(u) d u \\
& \left.+\int_{t}^{\sigma^{-1}(\delta(t))} Q(u) d u\right]
\end{aligned}
$$




\section{Hussain .A/Aqeel .F}

$$
\begin{gathered}
y(\delta(t)) \geq W(\delta(t))[1+P(\delta(t)) \\
+\int_{\alpha^{-1}(\delta(\delta(t)))}^{\delta(t)} R(u) d u \\
\left.+\int_{\delta(t)}^{\sigma^{-1}(\delta(\delta(t)))} Q(u) d u\right]
\end{gathered}
$$

Substituting the last inequality (3.2) in (2.3) we get

$$
\begin{aligned}
& W^{\prime}(t) \leq-\left[Q\left(\sigma^{-1}(\delta(t))\right)\left(\sigma^{-1}(\delta(t))\right)^{\prime}\right. \\
& \left.-R\left(\alpha^{-1}(\delta(t))\right)\left(\alpha^{-1}(\delta(t))\right)^{\prime}\right][1+P(\delta(t)) \\
& +\int_{\alpha^{-1}(\delta(\delta(t)))}^{\delta(t)} R(u) d u \\
& \left.+\int_{\delta(t)}^{\sigma^{-1}(\delta(\delta(t)))} Q(u) d u\right] W(\delta(t)) \\
& W^{\prime}(t)+\left[Q\left(\sigma^{-1}(\delta(t))\right)\left(\sigma^{-1}(\delta(t))\right)^{\prime}\right. \\
& \left.-R\left(\alpha^{-1}(\delta(t))\right)\left(\alpha^{-1}(\delta(t))\right)^{\prime}\right][1+P(\delta(t)) \\
& \left.+\int_{\alpha^{-1}(\delta(\delta(t)))}^{\delta(t)}+\int_{\delta(t)}^{\sigma^{-1}(\delta(\delta(t)))} Q(u) d u\right] W(\delta(t)) \\
& \quad(3.3)
\end{aligned}
$$

By Lemma 2.1, and the condition (3.1) the last inequality cannot has eventually positive solution, which is a contradiction. The proof is complete.

Corollary 3.2: Let $W(t)$ defined as in (2.2) and the assumptions H1-H3 hold, and there exist a continuous function $\delta(t)<t$ such that

$$
\begin{gathered}
{\left[Q\left(\sigma^{-1}(\delta(t))\right)\left(\sigma^{-1}(\delta(t))\right)^{\prime}\right.} \\
\left.\quad-R\left(\alpha^{-1}(\delta(t))\right)\left(\alpha^{-1}(\delta(t))\right)^{\prime}\right] \\
\geq \frac{1}{e \min _{t \geq t_{0}}\{t-\delta(t)\}} .
\end{gathered}
$$

Where $\alpha^{-1}(\delta(t))<t$ and $\sigma^{-1}(\delta(t))>t$, then every solution of equation (1.1) oscillates.

Proof. It is obvious that condition (3.4) implies that

$$
\begin{aligned}
\int_{\delta(t)}^{t}\left[Q\left(\sigma^{-1}(\delta(s))\right)\left(\sigma^{-1}(\delta(s))\right)^{\prime}\right. & \left.-R\left(\alpha^{-1}(\delta(s))\right)\left(\alpha^{-1}(\delta(s))\right)^{\prime}\right] d s \\
\geq & \frac{1}{e \min _{t \geq t_{0}}\{t-\delta(t)\}}(t-\delta(t)) \geq \frac{1}{e}
\end{aligned}
$$

Which leads to condition (3.1) holds. Hence according to Theorem 3.1 every solution of (1.1) oscillates.

\section{EXAMPLE}

In this section we give an example to illustrate the obtained results.

Example 4.1.Consider the impulsive neutral differential equation:

$$
\begin{aligned}
& {\left[y(t)-\frac{1}{9}\left(1+e^{-t}\right) y(t-2 \pi)\right]^{\prime}} \\
& +\frac{1}{9}\left(8-e^{-t}\right) y\left(t-\frac{5 \pi}{2}\right)-\frac{1}{9} e^{-t} y(t-2 \pi)=0 \\
& t \neq t_{k} \text { and } k=1,2, \ldots \\
& y\left(t_{k}^{+}\right)+b_{k} y\left(t_{k}\right)=a_{k} y\left(t_{k}\right), \quad t=t_{k} \text { and } k= \\
& 1,2, \ldots
\end{aligned}
$$

Where $a_{k}=\frac{2 k}{k+1}$ and $b_{k}=\frac{k}{k+1}$ we can see that

$$
a_{k}-b_{k}=\frac{2 k}{k+1}-\frac{k}{k+1}=\frac{k}{k+1}<1
$$

Let $\mathrm{P}(\mathrm{t})=\left\{\begin{array}{l}\left(\frac{1}{9}+\frac{1}{9} e^{-t}\right), t \neq t_{k} \\ \frac{1}{20 k}, \quad t=t_{k}\end{array}\right.$

Let $\delta(t)=t-\frac{9 \pi}{4}, \sigma^{-1}(\delta(t))=t+\frac{\pi}{4}$ and

$$
\alpha^{-1}(\delta(t))=t-\frac{\pi}{4} \text { to see condition } \boldsymbol{H 1}
$$

$$
\begin{aligned}
Q\left(\sigma^{-1}(\delta(t))\right)\left(\sigma^{-1}(\delta(t))\right)^{\prime} \\
\quad-R\left(\alpha^{-1}(\delta(t))\right)\left(\alpha^{-1}(\delta(t))\right)^{\prime} \\
=\frac{1}{9}\left(8-e^{-t-\frac{\pi}{4}}\right)-\frac{1}{9} e^{-t+\frac{\pi}{4}} \geq 0.5945 .
\end{aligned}
$$

Let $t_{k}=k, P\left(t_{k}^{+}\right)=P\left(k^{+}\right)=\lim _{t \rightarrow k^{+}} P(t)=$ $\lim _{t \rightarrow k^{+}}\left(\frac{1}{9}+\frac{1}{9} e^{-t}\right)=\left(\frac{1}{9}+\frac{1}{9} e^{-k}\right) \geq \frac{1}{9}$.

$$
\begin{aligned}
\left(a_{k}-b_{k}\right) P\left(t_{k}\right) & =\frac{k}{k+1} P(k)=\frac{k}{k+1} \frac{1}{20 k} \\
& =\frac{1}{20(k+1)} \leq 0.025, \text { and } \\
\frac{1}{\left(a_{k}-b_{k}\right)} P\left(t_{k}\right)= & \frac{k+1}{k} P(k)=\frac{k+1}{k} \frac{1}{20 k}=\frac{k+1}{20 k^{2}} \leq 0.1,
\end{aligned}
$$
so $\boldsymbol{H} 2$ holds.

And the condition $\boldsymbol{H 3}$ leads to

$$
\begin{aligned}
& \liminf _{\mathrm{t} \rightarrow \infty} \int_{\delta(t)}^{\mathrm{t}}\left[Q\left(\sigma^{-1}(\delta(\xi))\right)\left(\sigma^{-1}(\delta(\xi))\right)^{\prime}\right. \\
& \left.-R\left(\alpha^{-1}(\delta(\xi))\right)\left(\alpha^{-1}(\delta(\xi))\right)^{\prime}\right][1+P(\delta(\xi)) \\
& \left.+\int_{\alpha^{-1}(\delta(\delta(\xi)))}^{t} R(u) d u+\int_{\delta(\xi)}^{\sigma^{-1}(\delta(\delta(\xi)))} Q(u) d u\right] \mathrm{d} \xi \\
& =\frac{1}{9} \lim _{\mathrm{t} \rightarrow \infty} \int_{t-\frac{9 \pi}{4}}^{\mathrm{t}}\left[8-e^{-\xi-\frac{9 \pi}{4}}\right]\left[1+\frac{1}{9}+\frac{1}{9} e^{-\xi+\frac{9 \pi}{4}}\right. \\
& \left.+\frac{1}{9} \int_{\xi-\frac{10 \pi}{4}}^{\xi-\frac{9 \pi}{4}} e^{-u} d u+\frac{1}{9} \int_{\xi-\frac{9 \pi}{4}}^{\xi-\frac{8 \pi}{4}}\left(8-e^{-u}\right) d u\right] \mathrm{d} \xi \\
& \quad=11.3662>\frac{1}{e}
\end{aligned}
$$


Hence all conditions of theorem 3.1 hold, so all solutions of equation (3.1) are oscillatory. For instance the solution $y(t)=\left\{\begin{array}{l}\text { sint }, \quad t \neq t_{k} \\ 2+\frac{1}{k}, \quad t=t_{k}\end{array} \quad\right.$ is such a solution.

\section{REFERENCES}

1. A. d'onofrio: On pulse vaccination strategy in the SIR epidemic model with vertical transmission, Appl. Math. Lett., 18, 729-732, (2005).

2. G. Jiang and Q. Lu: Impulsive state feedback control of a predator-prey model, J. Comput. Appl. Math., 200, 193-207, (2007).

3. S. Nenov: Impulsive controllability and optimization problems in population dynamics, Nonl. Anal., 36, 881-890, (1999).

4. S. Ahmad and I. M. Stamova: Global exponential stability for impulsive cellular neural networks with time-varying delays, Nonlinear Anal. 69, 786-795, (2008).

5. M. B. Dimitrova and V. I. Donov: Oscillations of first order impulsive differential neutral constant delay equations with mixed coefficients, International Journal of Pure and Applied Mathematics, 83, No.3, 515-526, (2013).

6. M. B. Dimitrova and D. Mishev: Oscillation of the Solutions of Neutral Impulsive Differential -Difference Equations of First Order, Electronic Journal of Qualitative Theory of Differential Equations, 2005, No.16, 1-11, (2005).

7. Z. Geo and Z. Teng: Oscillation Criteria of Impulsive Neutral Parabolic Equations with Nonlinear Diffusion Coefficient, International Journal of Science, 11, No.2, 168-172, (2011).

8. R. P. Agarwal, S. R. Grace and D. O'Regan : Oscillation Theory for Difference and Functional Differential Equations, Kluwer Academic Publishers, (2000).

9. H. A. Mohamad and M. Y. Abdullah: Oscillations of First Order Neutral Differential Equations with Positive and Negative Coefficients, Baghdad Science Journal, 11, No.4, 1624-1629, (2014).
10. S. Pandian and Y. Balachandran, Some Oscillation Criteria for First Order Impulsive Neutral Differential Equation, International Journal of Mathematics and Scientific Computing, 1, No. 2, 22-25, (2011).

11. S. Pandian and G. Purushothaman, Oscillation of Impulsive Neutral Differential Equation with Several Positive and Negative Coefficients, J. Math. Comput. Sci., 2, No. 2, 241-254, (2012).

12. Y. Zhang, A. Zhao and J. Yan, Oscillation Criteria for Impulsive Delay Differential Equations, Journal of Mathematical Analysis and Applications, 205, 461-470, (1997).

13. Z. Lou, X. Lin, J. Shen and Z. Zou, Oscillation of Impulsive Neutral Differential Equations with Positive and Negative Coefficients, Indian Journal of pure and applied Mathematics, 31, No. 7, 753-766, (2000).

14. J. R. Graef, J. H. Shen and I. P. Stavroulakis, Oscillation of Impulsive Neutral Delay Differential Equations, Journal of Mathematical Analysis and Applications, 268, 310-333, (2002).

15. K. Guan and J. Shen, Oscillation Criteria for a First-Order Impulsive Neutral Differential Equation of Euler form Order, Computers and Mathematics with Applications (ELSEVIER), 58, 670-677, (2009).

16. J. Shen and Z. Zou, Oscillation Criteria for First-Order Impulsive Differential Equations with Positive and Negative Coefficients, Journal of Computational and Applied Mathematics, 217, 28-37, (2008).

17. G. Ye, J. Shen and J. Li, Periodic Boundary Value Problems Impulsive Neutral Differential Equations with Multi- Deviation Arguments, Computational and Applied Mathematics, 29, No. 3, 507-527, (2010). 


\title{
خاصية التذبذب لمعادلات تفاضلية محايدة متسارعة من الرتبة الاولى ذات المعاملات

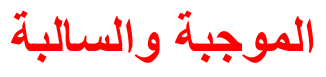

\author{
عقيل فالح جدوع \\ حسين علي محمد \\ جامعة بغداد \\ كلية العلوم للبنات كلية التربية للعلوم الصرفة إبن الهيثم \\ قسم الرياضيات
}

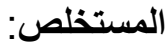

في هذا البحث حصلنا على الثروط الضرورية والكافية للتذبذب، ذلك أنه كل حل لمعادلات تفاضلية محايدة متسار عة خطية ذات تباطؤات متغيرة ومعاملات متغيرة يتذبذب.إن أغلب المؤلفين و الباحثين الذين درسوا ظاهرة التذبذب لمعادلات تفاضلية محايدة متسار عه كانوا قد اجروا در اسة لمعادلات ذات تباطؤات ثابتة ومعاملات ثابتة. على اية حال، إن نقاط التسارع هنا أكثر عمومية. لقد قدمنا مثنال توضيحي ليبر هن صحة النتائج. 